\title{
Voluntary Counseling and Testing (VCT) services and its role in HIVIAIDS prevention and management in Nepal
}

\author{
Preeti K. Mahato ${ }^{1}$, Peng $\mathrm{Bi}^{2}$, Teresa Burgess ${ }^{3}$ \\ ${ }^{1}$ Public Health Officer, Western Regional Health Directorate, Pokhara, Nepal; ${ }^{2}$ Professor; ${ }^{3}$ Senior Lecturer; Discipline of Public \\ Health, The University of Adelaide, Australia.
}

\begin{abstract}
HIV/AIDS has emerged as a serious epidemic in Nepal, concentrated amongst the most-at-risk populations. Voluntary Counseling and Testing (VCT) is a proven strategy for the prevention and management of HIV/AIDS especially in developing countries. Given that the utilization of VCT is low in Nepal, it is important to understand the barriers and challenges that Nepal is facing in the provision and utilization of these services. This study aimed to provide recommendations for policy makers to improve the utilization of VCT services in Nepal. A qualitative study of VCT health service providers and government officials was undertaken in 4 cities of Nepal. Sixteen interviews, three joint interviews and one focus group discussion were conducted, audio recorded and transcribed verbatim. Data were then analyzed using a thematic analysis framework and major themes were identified. Various barriers including sociocultural issues, geography, the presence of stigma and discrimination faced by people with HIV/AIDS and a lack of knowledge and awareness were identified as important factors limiting the utilization of VCT services. Current government efforts to improve VCT service provision, although promising, require ongoing development. Systemic barriers included the sustainability of the temporary NGO VCT services, lack of staff and training, inadequate monitoring and evaluation and limited access to services for people living in rural areas. To increase the sustainability and utilization of VCT services, the Government can intervene in a number of areas including the integration of VCT services with existing primary health care services and addressing ongoing education and quality assurance.
\end{abstract}

Keywords: HIV/AIDS, VCT, Challenges, Prevention and management, Nepal.

\section{Introduction}

HIV/AIDS has evolved as a serious epidemic in Nepal, concentrated amongst the most-at-risk population (MARP) groups: intravenous drug users (IDUs), female sex workers (FSWs), men who have sex with men (MSM) and labor migrants. ${ }^{1}$ In 2010, the adult prevalence of HIV infection was $0.33 \%$, decreasing from $0.5 \%$ in $2007 ;^{2}$ however, the prevalence of infection remains higher among subpopulations, especially in IDUs (Table 1). ${ }^{3}$ According to recent estimates, Nepal is home to approximately 50,200 people living with HIV and four out of every five infections in Nepal have occurred through sexual transmission. ${ }^{1}$ Though recent data shows stabilization of the epidemic among MARPs, the fact that many sex workers are IDUs, migrants, or both, increases the spread of HIV among these groups. A large portion of men who buy sex are married, which increases the possibility of spreading infection into the general population. ${ }^{1}$

Many people in Nepal are dying without knowing their HIV status despite the availability of treatment facilities. ${ }^{4}$ The government does not adequately address HIV prevention and treatment services as the current healthcare infrastructure is unable to provide sufficient services for those most at risk and there is a lack of service integration and referral systems. ${ }^{5}$ In addition, due to the lack of dissemination of health strategies and programs at various levels

\section{Practice Points}

- VCT services in Nepal face many barriers and challenges that result in low utilization of these services by the most at risk population (MARP) groups.

- Barriers and challenges are related to access, lack of knowledge and awareness of issues related to the prevention and management of HIV/AIDS; the presence of stigma and discrimination and issues related to sustainability.

- Integration with existing primary health care, sexual health and TB services offers the possibility of more referrals and easier access to services for people with HIV.

- Better collaboration between government, service providers and NGOs is necessary in order to increase and improve utilization of VCT services.

- Other locally focused strategies need to be developed apart from VCT services for the prevention of HIV/AIDS.

Correspondence: Professor Peng Bi, Discipline of Public Health, The University of Adelaide, SA 5005 Australia. E-mail: peng.bi@adelaide.edu.au. 
Table 1: HIV Prevalence rate among the most atrisk populations in Nepal $(\%)^{3}$

\begin{tabular}{|l|c|c|}
\hline \multicolumn{1}{|c|}{ MARP Groups } & $\mathbf{2 0 0 8}$ & $\mathbf{2 0 0 9}$ \\
\hline $\begin{array}{l}\text { Female sex workers (FSWs, } \\
\text { Kathmandu) }\end{array}$ & $2.2 \%$ & - \\
\hline $\begin{array}{l}\text { Male sex workers (MSWs, } \\
\text { Kathmandu) }\end{array}$ & - & $5.2 \%$ \\
\hline $\begin{array}{l}\text { Injecting drug users (IDUs, } \\
\text { Kathmandu) }\end{array}$ & - & $20.7 \%$ \\
\hline $\begin{array}{l}\text { Men who have sex with men } \\
\text { (MSM, Kathmandu) }\end{array}$ & - & $3.8 \%$ \\
\hline Migrants (West) & $0.8 \%$ & - \\
\hline Migrants (Far West) & & - \\
\hline
\end{tabular}

(village, district, zone, regional and central), ensuring delivery of quality health services in an equitable and sustainable manner is still a major challenge. ${ }^{6}$

The health system of Nepal is not decentralized, with most hospitals (private and government) located in urban areas. Although health services have been designed to ensure that the majority of people receive accessible, affordable treatment in public health facilities, people prefer the better quality services offered by the tertiary and private hospitals in urban areas, due to resource constraints and poor management in the rural public health system. ${ }^{7}$

A pivotal strategy for HIV/AIDS prevention and control, especially in developing countries, is Voluntary Counseling and Testing (VCT). ${ }^{8}$ VCT prevents transmission of the virus by combining personalized counseling with knowledge of one's HIV status, to motivate people to change their behaviors. ${ }^{9}$ Widespread use of HIV-1 VCT in less-developed countries has been effective in the prevention of HIV by enhancing behavior change so those infected seek care and support services. ${ }^{10,11}$ As an important and cost-effective HIV prevention strategy, with an increasing role in improving access to care and support, ${ }^{11}$ VCT services have been widely promoted in developing countries, as part of their primary health care package. ${ }^{12}$

HIV preventive programs in Nepal are mostly supported by External Development Partners (EDPs). ${ }^{3}$ There are 196 VCT centers operated by government, nongovernment organizations (NGOs) and international non -government organizations (INGOs). ${ }^{13}$ However concerns remain about VCT services including access, infrastructure, availability of trained professionals, quality of services, service operation hours and maintenance of privacy and confidentiality. ${ }^{14}$ Data show that $70 \%$ of the users of VCT are from the general population and migrants and there is a lower VCT utilization by certain MARP groups (such as IDUs and MSM), indicating the presence of barriers in accessing the VCT service among this population. ${ }^{3}$

A number of studies have investigated knowledge, acceptability and attitudes towards VCT services, ${ }^{15,16}$ but there are few studies which identify the barriers and challenges faced in providing VCT services in developing countries such as Nepal. ${ }^{17,18,19}$ In spite of numerous efforts by the Nepali Government, HIV prevention and treatment services are often not reaching MARP groups, which may be due to gaps in planning and implementation by policy makers. ${ }^{14}$ Therefore, an understanding of the difficulties faced in the provision and utilization of these services, especially by the MARP groups, is urgent. This study focuses on the barriers and challenges faced in providing the VCT and suggests some ways in which services can be improved.

\section{Materials and methods}

\section{Study design}

A qualitative approach explored barriers and challenges in the provision and utilization of VCT services in four cities of Nepal: Kathmandu, Pokhara, Dharan and Janakpur, all of which have high percentages of IDUs, FSWs, MSM and labor migrants. ${ }^{1}$ A high percentage of MARP groups live in the Kathmandu valley and the majority of VCT centers are located in urban areas, with nearly $50 \%$ of HIV infections recorded in Pokhara, Dharan and Janakpur. ${ }^{3}$

Ethical clearance was obtained from The University of Adelaide Human Research Ethics Committee and permission and approval to conduct the research was obtained from the National Centre for AIDS and STD Control (NCASC), Kathmandu, Nepal. The research was carried out from March 2011 until August 2011.

\section{Selection and recruitment of participants}

A purposive sampling method targeted particular groups of VCT service providers as well as decision-makers, to identify and compare their differing perspectives. Purposive sampling allows the selection of specific participants, events or settings intentionally because of detailed information that they can provide which may not be available using other sources. ${ }^{20}$ Table 2 details the final sample of twenty participants:

i. Government officials working at the NCASC and District AIDS Co-ordination committees (DAAC)

ii. VCT service providers from government and non-government sectors, (managers or counselors)

iii. HIV negative representatives from the general community

Male and/or female participants who were $>25$ years of age were contacted by telephone and asked to participate in study. Meetings were organized at the convenience of participants.

Interviews and Focus Group Discussions (FGD)

Face-to-face interviews were undertaken using a semistructured interview schedule, which was designed to explore the current functioning of VCT services. The questions were developed using evidence from the lit- 
Table 2: Interviews/focus groups

\begin{tabular}{|l|c|c|c|c|}
\hline Type of interviews & \multicolumn{1}{|c|}{ Pokhara } & Kathmandu & Dharan & Janakpur \\
\hline & VCT service providers & 2 & 2 \\
\hline Individual & 3 & 4 & & \\
\hline Joint & 2 & 1 & & \\
\hline FGD & 1 & & \\
\hline & \multicolumn{3}{|l}{} \\
\hline Individual & Government personnel & \\
\hline & \multicolumn{3}{|l}{} \\
\hline Individual & Representatives from the general population & & \\
\hline
\end{tabular}

erature which considered the barriers and challenges faced by the VCT services. We wished to know if these were reflected in the Nepali context and if any other barriers or challenges could be identified in providing and accessing these VCT services. Sixteen interviews, 3 joint interviews and 1 FGD (with four participants) were conducted over 5 weeks in four cities. The interviews and FGD, were conducted in Nepali and were audio recorded with the permission of the participants and downloaded to a password protected computer and transcribed verbatim.

\section{Data analysis}

Data were analyzed using the thematic analysis framework suggested by Braun and Clarke. ${ }^{21}$ Analysis began with the process of translation of the interviews and focus groups into English by PM. All transcripts were read a number of times and coded systematically by PM using both a deductive approach (identifying specific issues explored in the interview schedules) and an inductive approach, to identify any new issues arising which had not been previously recognized. 'QSRNVivo 9",22 was used to assist with data analysis. A number of categories were identified which were refined and named as major themes. The reliability of the codes was checked by PB and TB. Figure 1 describes the method and steps of the thematic analysis.

\section{Results}

A number of themes emerged from the data in the areas of barriers and challenges. Barriers were perceived as broad elements impacting on both service providers and the community whilst challenges were specifically systems related issues requiring to be addressed at the government/service management level.

\section{Barriers}

\section{Stigma and discrimination about HIV infections}

Almost all participants reported the presence of stigma and discrimination in Nepali society, especially in rural areas. Both VCT providers and NCASC personnel noted that being recognized as an HIV positive patient was a barrier for the utilization of VCT services.

"HIV patients are still not accepted in our society. The presence of stigma and discrimination in society has caused fear among the HIV patients, so they don't feel secure in utiliz- ing VCT services, and they also don't undertake health seeking behaviours" (Government VCT provider participant)

Some service providers also noted that the voluntary uptake of VCT services by MARP groups was low and these groups do not utilize VCT services even when they find themselves at risk.

\section{Knowledge and awareness among the general commu-} nity

Some participants believed that awareness regarding HIV/AIDS has been increasing among the general population, however they also stated there was a lack of knowledge about risky behaviors, safe sex and importance of VCT services. A majority of the participants still felt there was a need to increase awareness in the general community regarding most aspects of HIV/ AIDS prevention and management. A lack of education around normalizing HIV/AIDS through discussion with the general population and positive preventive messages was also identified by participants as a key barrier. This problem was compounded by lack of information about where to go to check HIV status. Participants generally believed that there are still misconceptions among people regarding the mode of transmission of HIV/AIDS.

"There is lack of awareness among rural people regarding HIV/AIDS, its mode of transmission as well as where to utilize the services aimed for HIV patients" (NGO VCT provider participant)

Participants focused on a need to view HIV as a chronic manageable disease rather than a terminal disease.

Geographical location of the services

Service providers noted that the geographical location of services and the location of most VCT services in urban areas was seen as an important barrier for people in rural areas.

\section{"If VCT services were available from health posts and sub health posts in rural areas, only then would they be accessible to all" (NGO VCT provider participant)}

NGO VCT services employ outreach educators who create awareness among the people in rural areas and motivate them to utilize VCT services, which is an 


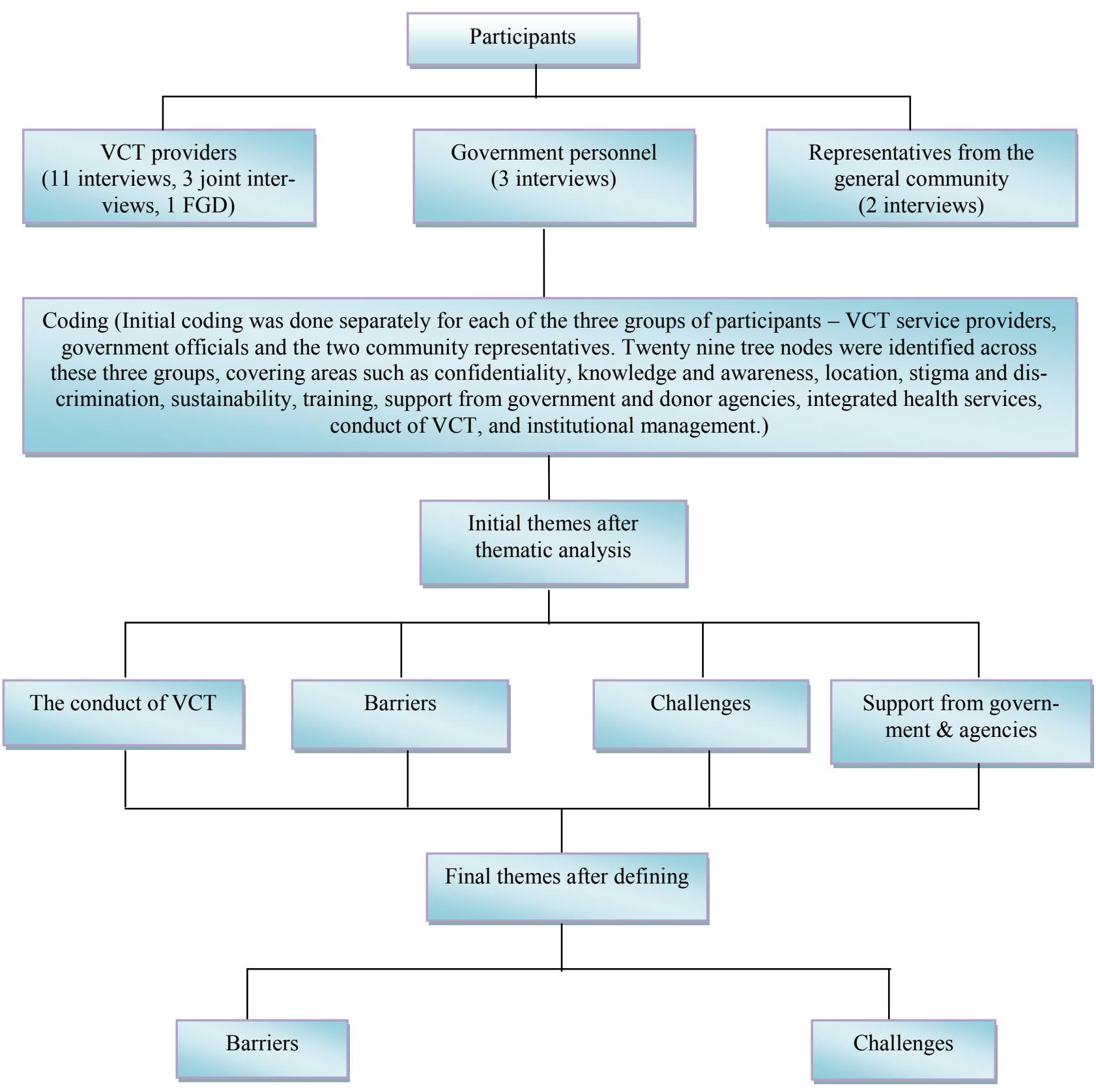

Figure 1: Coding of interview/focus group data

effective prevention mechanism. Providers suggested that the expansion of mobile clinics, outreach educators and home-based care in rural areas may help to address these issues. While NCASC personnel also felt a need to expand the services to rural areas, they noted the mountainous topography of Nepal, which keeps rural areas isolated due to lack of an effective road system and inhibits effective access and development.

\section{Program Management Issues}

A number of service providers identified that a lack of uniformity in the monitoring, supervision and reporting from NCASC made managing VCT programs difficult. Lack of manpower due to high turnover (related to better job opportunities, study opportunities etc.) and unfilled, vacant posts were identified as major problems by the policy makers.

\section{Challenges}

Staff Training and Quality Assurance

Almost all service providers noted a lack of adequate staff training available from the NCASC, especially counselors, and that the training was not provided at the beginning of year when it would be most useful.

"There is not regular training given to the staff especially the counselors." (NGO VCT provider participant)

NCASC personnel believed that the NCASC was not directly responsible for providing training to the NGO run VCT centers, though they provide training to the government run VCT centers. 


\section{Service Sustainability}

Service providers from donor-run VCT centers expressed concerns regarding sustainability, as these services were supported by short term funding from donor agencies. They suggested that the government should increase its involvement in the management of funding and programs to ensure sustainability of available VCT services.

"There is a lack of sustainability of HIV program run by NGOs. The government should take the initiative to ensure that the programs are run for the long term" (NGO VCT provider participant)

\section{Availability of Staff}

Both the donor-run and government-owned service providers identified a need to increase the number of trained personnel. NCASC participants accepted that there is a shortage of trained staff in VCT centers and noted that they are in the process of increasing training of staff.

Staff working at VCT centers also noted that they faced discrimination from the broader society as working with groups such as FSW, MSM, IDU is viewed negatively, causing problems for service providers, especially for the outreach staff in educating the local people.

"We work with the FSWs, who are considered as being immoral and are stigmatized and discriminated against. Many times they think that we also have the same background as the FSW and their clients" (NGO VCT provider participant)

\section{Integrated health services}

The development of integrated health services including VCT centers was identified by service providers as helpful for patients as they could then utilize other services to provide care and support for HIV positive patients. Four participants particularly noted the difficulties caused for patients by having to refer them to other hospitals because of a lack of integrated services.

The government has done a lot for patients suffering from diseases like TB and leprosy to fight against them; HIV/AIDS patients should be treated in the same way by building centres for these patients and making the VCT services available to the general public. (NGO VCT provider participant)

\section{Discussion}

This research has identified that VCT services in Nepal face many barriers and challenges that result in low utilization of those services particularly by MARP groups. Four main contributing factors were identified:

Stigma \& discrimination related to HIV/AIDS in society The presence of HIV/AIDS related stigma and discrimi- nation in Nepal acts as an important barrier in the utilization of VCT services, especially in rural areas. Fear of being recognized as HIV positive prevents people from accessing VCT services, resulting in low voluntary uptake of these services. This finding was consistent with several other studies reporting the presence of HIV/AIDS related stigma and discrimination and the fear of being recognized among patients. ${ }^{16,18,19}$ Negative social and cultural views towards FSWs, IDU and MSM in Nepali society hinders utilization of HIV prevention and treatment services. ${ }^{23}$ Talking about sex is considered disrespectful and impolite in Nepal and as a consequence of these socio cultural norms, efforts to mitigate the impact of HIV/AIDS is challenging, as well as being responsible for diminishing access to preventive services. ${ }^{14}$ This stigma extends to the workforce - people working with MARP groups and people with HIV experience discrimination from the broader public which has implications for the recruitment and retention of staff in an area where workforce is already an issue.

Participants suggested that increasing positive prevention and starting to talk about HIV in family and society groups would have a positive influence on the number of people accessing VCT services. These suggestions reflect other studies which found that HIV discussion among community members has a positive influence on people's attitudes. ${ }^{24}$ Positive prevention includes experience sharing by People Living with HIV (PLHIV) and encouraging access to VCT, Anti Retroviral Therapy (ART) and other services. ${ }^{3}$

Knowledge and awareness among the general community and MARP groups

A lack of knowledge and awareness regarding HIV/ AIDS, its mode of transmission and the importance of VCT services among the general population was identified by participants, reflecting other studies with similar findings. ${ }^{16}$ The UN General Assembly Special Session (UNGASS) report also points out that though the general awareness of HIV/AIDS is high, a comprehensive awareness on HIV and AIDS is comparatively low among some of the MARPs. ${ }^{3}$

A key driver for this research was the decrease in VCT utilization with the rate per VCT center decreasing from 10 per week in 2008 to 7 per week in $2009 .{ }^{3}$ Using educators from organizations such as PLHIV has proven to be very effective in increasing knowledge regarding HIV and VCT services, promoting safe sex and encouraging behavior change by acting as highly effective outreach educators and community mobilizers. ${ }^{3}$ Our study found outreach and community mobilizers to be more effective than urban based centers in increasing the knowledge and awareness related to HIV/AIDS. Increasing the number of outreach educators to the rural areas could be helpful in increasing knowledge related to HIV/AIDS. A WHO report on HIV testing and counseling also suggests a need for mobile and outreach services for house to house visits in high prevalence areas, and providing VCT services to those who are most unlikely to seek testing on their own. ${ }^{25}$ 
Access

This research highlighted access related issues especially for labor migrants who mostly reside in remote areas with few resources. ${ }^{3}$ They cannot therefore access the urban location of most VCT centers and many high risk behaviors that expose people to HIV are related to geographical location. ${ }^{14,17}$ Participants noted too, the difficult geographical topography which creates problems in providing adequate prevention and care facilities in rural areas. ${ }^{14}$

Service providers suggested the provision of government funding to expand services to rural areas and the provision of mobile services to visit remote areas. NCASC officials noted that the government is in the process of scaling up VCT services to the level of PHC in accordance with one of the six national program areas focusing on increasing the availability and access to prevention services. ${ }^{3}$ The provision of a mobile van VCT service to rural areas to improve access to potential clients was recommended by a study in Uganda. ${ }^{26}$ In conjunction with other sexual and reproductive health services, it provides a greater level of privacy for people seeking HIV services, and so they may be more willing to utilise them. ${ }^{27,5}$

\section{Sustainability of VCT services}

The sustainability of VCT programs has been identified as a challenge by $\mathrm{UNGASS}^{3}$ and this research highlighted the need for the government to improve its service provision capacity, particularly in the areas of staff recruitment, retention and training. The shortage of trained staff was noted as a major challenge in the dayto-day running of VCT centers and this was also identified by the WHO; who suggested sufficient number of counselors be recruited from various backgrounds including people living with HIV/AIDS, community volunteers and a new cadre of paid counselors. ${ }^{28}$ Short term funding was also identified as an issue, with providers suggesting gradually shifting VCT program and funding management to national institutions for long term sustainability. ${ }^{3}$ Linking VCT programs more closely with existing PHC services could allow greater support for VCT counselors, both on a personal and professional level.

A majority of participants identified the importance of integrating health services, allowing patients to utilize VCT services along with other related services such as STI, ART, TB and family planning. Linking HIV testing with sexual and reproductive health makes it available to many people who may not have access to VCT services and offering VCT services to clients seeking STI care can provide tailored counseling based on HIV status. ${ }^{5}$ The government's current strategy of expanding DACC in every district and establishing VACC in rural areas will help to increase community participation and increase the co-ordination between national and grass root level centers. The Nepali government is in the process of making provider initiated testing and counseling (PITC) available from the TB, STI and ANC clinics, in order to identify the HIV infected people that require VCT services.
This research has a number of limitations. The issues have been considered from the perspective of service providers and policy makers with no input from the patients who utilize these services who may have different views from the service providers. Another limitation is the time constraints in data collection that restricted the number of interviews. Our research is not meant to be representative of the whole Nepali population as most of the participants were from VCT centers located in urban areas, but as most VCT services are located in urban areas, the results are useful in informing strategies to address barriers to service and for future policy development.

\section{Conclusion}

This study identifies a number of barriers and challenges for VCT services in Nepal. In order to increase the utilization of the VCT services, the government could intervene in a number of areas: 1) the integration of VCT services with sexual health and primary health care offers the possibility of more referrals and easier access to services for people with HIV, however staffing issues (including recruitment, retention and training) need to be addressed; 2) better collaboration between government, service providers, and NGOs; 3 ) Identification of alternative strategies for the prevention and management of HIV/AIDS apart from the VCT services, taking into consideration the difficulties that VCT services are facing in terms of access, sustainability and stigma and discrimination in Nepali society.

\section{References}

1. United States Agency for International Development. HIV/AIDS Health Profile for Nepal. http:// www.usaid.gov/nepal (accessed March 2013)

2. National Centre for AIDS and STD Control. Factsheet No 1: HIV Epidemic Update of Nepal. Teku: National Centre for AIDS and STD Control, Ministry of Health and Population, 2011.

3. UNGASS. Country Progress Report: Nepal. 2010. Available: http://www.unaids.org/en/ dataanalysis/monitoringcountrypro gress/2010progressreportssubmitted bycountries/ (accessed June 2013)

4. Kshetry R. Health- Nepal: meeting millennium development goal on HIV/AIDS - a dream? http://www.ipsnews.net/2008/10/health-nepalmeeting-mdg-on-hiv-aids-a-dream/ (accessed June 2013).

5. International Planned Parenthood Federation (IPPF). Voluntary Counselling and Testing: A gateway to linking HIV and sexual and reproductive health. London: IPPF, 2007.

6. Devkota B. Effectiveness of Essential Health Care Services Delivery in Nepal. J Nepal Health Res Counc 2009; 6:74-83.

7. WHO Regional Office For South-East Asia. 
Country Health System Profile: Nepal. http:// www.nep.searo.who.int/EN/Section5.htm (accessed June 2013)

8. World Health Organization. UNAIDS/WHO Policy Statement on HIV Testing. Geneva: WHO, 2004.

9. Denison JA, O’Reilly KR, Schmid GP, Kennedy CE \& Sweat MD. HIV voluntary counseling and testing and behavioral risk reduction in developing countries: a meta-analysis, 1990-2005. AIDS Behavior 2008; 12:363-73.

10. Coates TJ. Efficacy of voluntary HIV-1 counselling and testing in individuals and couples in Kenya, Tanzania, and Trinidad: a randomised trial. Lancet 2000; 356:103-12.

11. UNAIDS. The impact of Volunatry Counselling and Testing: A global review of the benefits and challenges. Geneva: UNAIDS, 2001.

12. Coovadia HM. Access to voluntary counseling and testing for HIV in developing countries. Ann N Y Acad Sci 2000; 918:57-63.

13. National Centre for AIDS and STD Control. Factsheet No 3: HIV Testing and Counselling Services in Nepal. Teku: National Centre for AIDS and STD Control, Ministry of Health and Population, 2010.

14. Wasti SP, Simkhada P, Randall J \& van Teijlingen E. Issues and Challenges of HIV/AIDS Prevention and Treatment Programme in Nepal. Glob J Health Sci.2009; 1:62-72.

15. Hesketh T, Duo L, Li H, Tomkins AM. Attitudes to HIV and HIV testing in high prevalence areas of China: Informing the introduction of voluntary counselling and testing programmes. Sex Transm Infect 2005;81:108-12.

16. Kalichman SC, Simbayi LC. HIV testing attitudes, AIDS stigma, and voluntary HIV counselling and testing in a black township in Cape Town, South Africa. Sex Transm Infect 2003; 79:442-7.

17. Bwambale FM, Ssali SN, Byaruhanga S, Kalyango JN, Karamagi CAS. Voluntary HIV counselling and testing among men in rural western Uganda: Implications for HIV prevention. $B M C$ Public Health. 2008; 8:263-74.
18. Mahendradhata Y, Ahmad RA, Lefevre P, Boelaert M \& Stuyft PVD. Barriers for introducing HIV testing among tuberculosis patients in Jogjakarta, Indonesia: A qualitative study. BMC Public Health. 2008; 8:385-94.

19. Meiberg AE, Bos AE, Onya HE and Schaalma HP. Fear of stigmatization as barrier to voluntary HIV counseling and testing in South Africa. East Afr J Public Health 2008; 5:49-54.

20. Liamputtong P, editor. Research Methods in Health. Melbourne: Oxford University Press, 2010 .

21. Braun V, Clarke V. Using thematic analysis in psychology. Qual Res Psychol 2006; 3:77-101.

22. NVivo 9. NVivo qualitative data analysis software; QSR International Pty Ltd. Version 9, 2010 .

23. Beine DK, editor. Ensnared by AIDS: cultural contexts of HIV/AIDS in Nepal. Kathmandu: Mandela Book Point, 2003.

24. Mugisha E, van Rensburg GH, Potgieter E. Factors Influencing Utilization of Voluntary Counseling and Testing Service in Kasenyi Fishing Community in Uganda. $J$ Assoc Nurses AIDS Care 2010; 21:503-11.

25. World Health Organization. Service delivery approaches to HIV testing and counseling (HTC): a strategic HTC policy framework. Geneva: WHO, 2012.

26. Narathius A. Feasibility study of the mobile van for voluntary counseling and testing (VCT) for HIV/AIDS. Final report submitted to the Uganda Program for Human and Holistic Development (UPHOLD), Kampala, Uganda. Kampala: Department of Social Work \& Social Administration, Makerere University, 2004.

27. Bradley H, Bedada A, Tsui A, Brahmbhatt H, Gillespie D, Kidanu A. HIV and family planning service integration and voluntary HIV counseling and testing client composition in Ethiopia. AIDS care. 2008; 20:61-71.

28. World Health Organization. Increasing access to HIV testing and counselling: Report of a WHO Consultation. Geneva: WHO, 2002. 